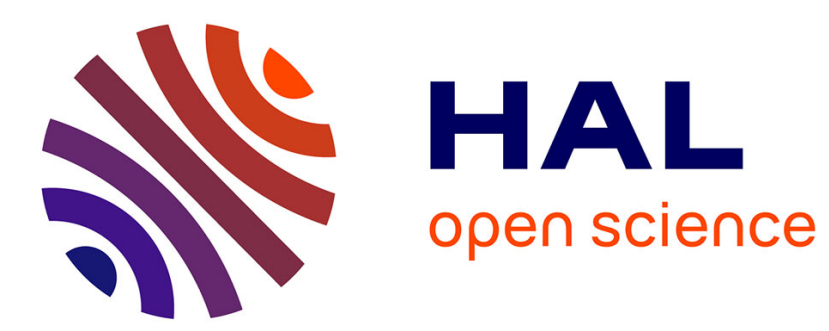

\title{
SPECTROSCOPIE ASTRONOMIQUE PAR TRANSFORMATION DE FOURIER
}

\author{
J. Connes, Pierre Connes, J. Maillard
}

\section{To cite this version:}

J. Connes, Pierre Connes, J. Maillard. SPECTROSCOPIE ASTRONOMIQUE PAR TRANSFORMATION DE FOURIER. Journal de Physique Colloques, 1967, 28 (C2), pp.C2-120-C2-135. 10.1051/jphyscol:1967224 . jpa-00213202

\section{HAL Id: jpa-00213202 https://hal.science/jpa-00213202}

Submitted on 1 Jan 1967

HAL is a multi-disciplinary open access archive for the deposit and dissemination of scientific research documents, whether they are published or not. The documents may come from teaching and research institutions in France or abroad, or from public or private research centers.
L'archive ouverte pluridisciplinaire HAL, est destinée au dépôt et à la diffusion de documents scientifiques de niveau recherche, publiés ou non, émanant des établissements d'enseignement et de recherche français ou étrangers, des laboratoires publics ou privés. 


\title{
SPECTROSCOPIE ASTRONOMIQUE PAR TRANSFORMATION DE FOURIER
}

\author{
J. CONNES \\ Observatoire de Meudon, 92-Meudon, France \\ P. Connes et J. P. Malllard \\ Laboratoire Aimé Cotton, CNRS, 92-Bellevue, France
}

\begin{abstract}
Résumé. - De nouveaux progrès ont été réalisés dans la production de spectres planétaires par spectroscopie de Fourier, principalement par l'application d'une méthode de modulation réduisant l'effet de la turbulence. Un autre facteur important est la transmission immédiate de l'interférogramme à un ordinateur qui permet d'avoir un premier résultat au cours de la même nuit d'observation. Une limite de résolution de $0,3 \mathrm{~cm}^{-1}$ a été atteinte sur Jupiter, et de $0,07 \mathrm{~cm}^{-1}$ sur Vénus dans les fenêtres du proche infrarouge $\left(8000,6000\right.$ et $\left.4300 \mathrm{~cm}^{-1}\right)$.
\end{abstract}

Abstract. - Improved planetary spectra have been produced by Fourier Spectroscopy. The two most important factors have been the use of a new modulation method which reduces considerably the effects of atmospheric turbulence, and a fast transmission system which enables the experimenter to get preliminary results during the observation night. The spectral resolution is $0.3 \mathrm{~cm}^{-1}$ for Jupiter and $0.07 \mathrm{~cm}^{-1}$ for Venus, in the near infrared windows $(8000$, 6000 and $4300 \mathrm{~cm}^{-1}$ ).

1. Introduction. - Le principe de la méthode d'enregistrement pas à pas ainsi que les premiers résultats donnés par notre interféromètre dans le proche infrarouge ont été décrits ailleurs [1]. Nous ne ferons que les rappeler brièvement ici :

Dans un enregistrement pas à pas, le chariot mobile de l'interféromètre stationne sur chacune des valeurs de la différence de marche pour lesquelles un échantillon de l'interférogramme est désiré ; le flux sortant de l'interféromètre est mesuré par intégration. Le chariot est alors déplacé aussi rapidement que possible jusqu'au point suivant. Les déplacements sont mesurés par comptage de franges d'une raie monochromatique, et les positions d'arrêt définies par asservissement au moyen $\mathfrak{d} \mathfrak{u}$ même signal de franges. Ce système déjà décrit - a été utilisé sans modifications dans le présent travail.

Les avantages par rapport à la méthode usuelle d'enregistrement continu sont les suivants :

1) La très grande précision avec laquelle la différence de marche peut être contrôlée entraîne une précision remarquable de la fonction d'appareil, qui peut avoir des maximum secondaires plus réduits et un contraste supérieur à ceux donnés par les meilleurs réseaux ou étalons Fabry-Perot.

2) Le nombre minimum de points permis par le théorème de l'échantillonnage peut être mesuré, ce qui est une nécessité dans le cas de spectres étendus, ou plus exactement contenant un grand nombre $M$, d'éléments spectraux $(M=\Delta \sigma / \delta \sigma$, avec $\Delta \sigma$ domaine spectral, et $\delta \sigma$ limite de résolution, ou largeur instrumentale).

3) Le temps d'intégration sur chaque point peut être variable, ce qui permet une compensation facile de l'effet des fluctuations d'intensité de la source.

4) L'enregistrement peut être interrompu et repris à volonté.

Les performances de l'interféromètre ont été vérifiées sur des sources de laboratoire. Une largeur instrumentale de $0,07 \mathrm{~cm}^{-1}$, conforme à ce que l'on peut attendre de la différence de marche maximum de l'appareil, qui est égale à $11 \mathrm{~cm}$, a été obtenue en émission et en absorption. Un rapport signal sur bruit supérieur à $10^{4}$ a été mesuré sur des raies d'émission. Le défaut de reproductibilité des intensités pour un spectre d'absorption a été trouvé inférieur à $1 / 400$, et le défaut de reproductibilité de position des raies inférieur à 1/500 de la largeur instrumentale ( ${ }^{1}$ ).

(1) Un nouvel interféromètre de même conception générale, mais permettant d'atteindre une différence de marche maximum voisine de $2 \mathrm{~m}$ et une limite de résolution de $5 \times 10^{-3} \mathrm{~cm}^{-1}$ est décrit par J. Pinard dans une autre communication à ce même Colloque [11]. 
Cet interféromètre, construit en collaboration avec le Jet Propulsion Laboratory (Pasadena, Californie) $\left({ }^{2}\right.$ ) est destiné à des études astronomiques, principalement planétaires. Au cours d'observations faites à Steward Observatory (Arizona) et à l'Observatoire de Saint Michel en Haute Provence, il a permis d'enregistrer des spectres de Vénus et Mars [1] avec une limite de résolution de l'ordre de $1 \mathrm{~cm}^{-1}$ dans les fenêtres atmosphériques du proche infrarouge $(1,25 \mu, 1,6 \mu$ et $2,2 \mu)$. Le gain en pouvoir de résolution par rapport aux mêmes spectres enregistrés par des méthodes classiques (spectromètre à réseau balayant le domaine spectral) était de l'ordre de 10 ; dans le cas de Mars, avait de plus été obtenue une importante amélioration du rapport signal sur bruit. L'analyse de ces spectres est en cours au Jet Propulsion Laboratory.

En raison de difficultés particulières aux observations astronomiques, et dues entièrement à la turbulence atmosphérique, le pouvoir de résolution maximum de l'appareil n'avait pas, jusqu'ici, été atteint sur les planètes. Le présent article décrit les dernières améliorations du système qui ont démontré leur efficacité au cours d'observations de Jupiter (janvier 1966) et de Vénus (mai-juin-juillet 1966) ( ${ }^{3}$ ) à l'Observatoire de Haute Provence du Centre National de la Recherche Scientifique. Les modifications de l'interféromètre ont été exécutées au Laboratoire Aimé Cotton. Tous les calculs ont été effectués par le Centre de Calcul Numérique de l'Observatoire de Meudon.

2. Turbulence atmosphérique et spectroscopie de Fourier. - Les perturbations apportées par 1'atmosphère terrestre peuvent (en simplifiant quelque peu) être groupées en trois catégories :

1) Les fluctuations de transparence achromatiques - au moins en première approximation et dans un domaine spectral pas trop étendu - dues essentiellement aux nuages légers à travers lesquels les observations doivent - trop souvent - être faites.

Ces variations peuvent être très importantes. Mais elles sont faciles à mesurer au moyen d'un récepteur supplémentaire, et leur compensation - par variation du temps d'intégration ou l'emploi d'autres dispositifs permettant d'enregistrer un rapport - est facile et ne mérite pas d'être étudiée en détail.

(2) Et transporté en France grâce au contrat AF-61 (052) 842, European Office of Aerospace Research, United States Air Force.

(3) Nous avons inclu dans la rédaction du présent article certains résultats obtenus sur Vénus peu après le Colloque; l'équipement était le même que celui utilisé sur Jupiter en janvier.
2) Les fluctuations de transparence fonction de la longueur d'onde à l'intérieur du domaine spectral étudié, pour lesquelles aucune compensation n'est possible.

Dans l'infrarouge, la cause la plus importante de fluctuations chromatiques de transparence est la variation de la teneur en vapeur d'eau de l'atmosphère, qui entraîne une fluctuation de profondeur de toutes les raies d'absorption correspondantes. Cet effet peut être réduit en limitant au moyen d'un filtre le domaine spectral à une "fenêtre " de transparence atmosphérique, pour laquelle les raies de la vapeur d'eau sont relativement peu intenses; c'est ce que nous avons fait jusqu'ici. Cependant, les filtres utilisés ne coïncidaient pas exactement avec les fenêtres et dans plusieurs cas sur le côté de la bande passante étaient incluses de nombreuses raies de la vapeur d'eau dont les profondeurs d'absorption allaient jusqu'à $100 \%\left({ }^{4}\right)$. Même dans ces conditions, l'effet est négligeable par rapport à ceux qui seront étudiés plus loin.

Comme l'observation à partir du sol des régions spectrales où l'absorption de la vapeur d'eau est presque totale, est dépourvue d'intérêt en astronomie stellaire ou planétaire, les conséquences pratiques de l'effet discuté ici sont peu importantes et ne limitent pas jusqu'à présent, le gain considérable donné par le principe multiplex.

3) Les fluctuations d'indice - ou turbulence proprement dite - causent d'une part une fluctuation dans le flux total collecté par le télescope, ou scintillation, qui peut être considérée comme sensiblement indépendante de la longueur d'onde dans un domaine spectral d'étendue modérée $\left({ }^{5}\right)$, tel que celui limité par une "fenêtre » infrarouge. Sa compensation est donc facile. Mais d'autre part, la structure du faisceau reçu est profondément modifiée. Aucune de ses sections droites n'est stable ni homogène. Seules les images du miroir primaire ont un contour fixe ; mais elles ne sont pas uniformément éclairées (phénomène des ombres volantes).

(4) Voir en particulier le spectre de Mars [1, p. 908].

(5) Ce ne serait plus vrai si l'on considérait, par exemple, l'ensemble du domaine compris entre 1 et $12 \mu$. Mais les récepteurs photoconducteurs ont, pour des raisons fondamentales, des domaines de sensibilité optimum limités. Si l'on cherchait à couvrir, en un seul enregistrement, l'ensemble de cette région spectrale - tout en obtenant le meilleur rapport signal sur bruit possible - il faudrait nécessairement disposer à la sortie de l'interféromètre un système disperseur et plusieurs récepteurs en parallèle, chacun d'eux étant choisi pour une fenêtre particulière. 
Or, le faisceau possédant cette structure doit traverser un système optique quelque peu complexe, comportant un grand nombre de miroirs dont le pouvoir réflecteur n'est pas rigoureusement uniforme, ainsi qu'une lame séparatrice, pour aboutir sur un ou plusieurs récepteurs tels que des cellules au sulfure de plomb dont la sensibilité est très peu uniforme. Les défauts d'homogénéité du faisceau donnent alors naissance à des fluctuations supplémentaires du signal dont la compensation est beaucoup plus difficile que celle de la scintillation pure car elles ne sont pas corrélées pour deux récepteurs différents. Ce phénomène se produit même si - comme il est correct - c'est l'image du miroir et non celle de la planète qui est projetée sur les récepteurs.

Bien que ces fluctuations soient assez faibles (de l'ordre de quelques pour cent du signal moyen avec une bande passante de $1 \mathrm{~Hz}$ ) elles ont constitué jusqu'ici la principale difficulté pour l'application astronomique de la spectroscopie de Fourier, alors qu'elles peuvent passer relativement inaperçues dans d'autres types de mesures $\left({ }^{6}\right)$.

Cette difficulté vient du principe même de la méthode. Le signal enregistré qui donne l'interférogramme, résulte en effet de la contribution de tous les éléments spectraux simultanément observés; c'est précisément ce qui entraîne le multiplexage du détecteur et permet le gain fondamental égal à un facteur $M$ sur le temps de mesure par rapport aux méthodes classiques d'exploration du spectre par balayage. Chaque élément spectral produit dans l'interférogramme une sinusoïde élémentaire dont l'amplitude est - en supposant le spectre rectangulaire pour simplifier -

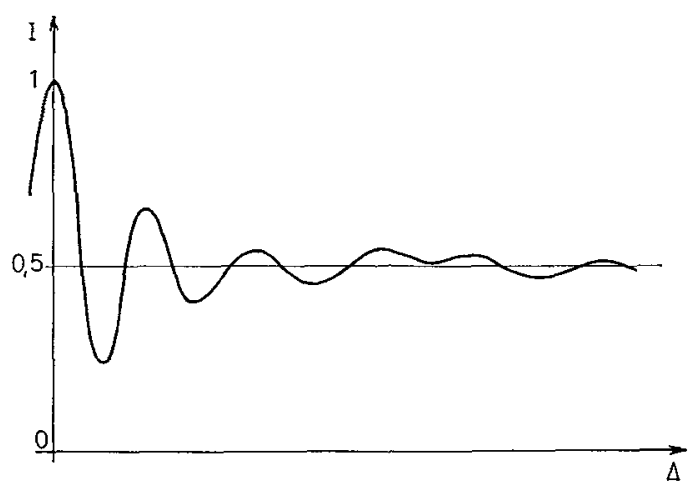

Fig. 1. - Aspect typique d'un interférogramme.

(6) En particulier, le temps d'intégration utilisé normalement en photométrie astronomique est de quelques dizaines de secondes; nous verrons plus loin que le temps d'intégration par point pour un enregistrement d'interférogramme à haute résolution peut être d'une fraction de seconde. égale à la fraction $1 / M$ de celle du pic central correspondant à la différence de marche zéro.

En conséquence, l'aspect habituel d'un interférogramme est celui dọné par la figure 1 : lorsque la différence de marche augmente, les variations d'intensité de l'interférogramme deviennent très petites vis-àvis de sa valeur moyenne; or ces variations d'intensité constituent les détails utiles qui doivent être correctement enregistrés. Toute fluctuation accidentelle, même très faible, de la valeur moyenne, peut causer une déformation considérable du spectre calculé. Donnons un exemple très schématique (Fig. 2). Soit

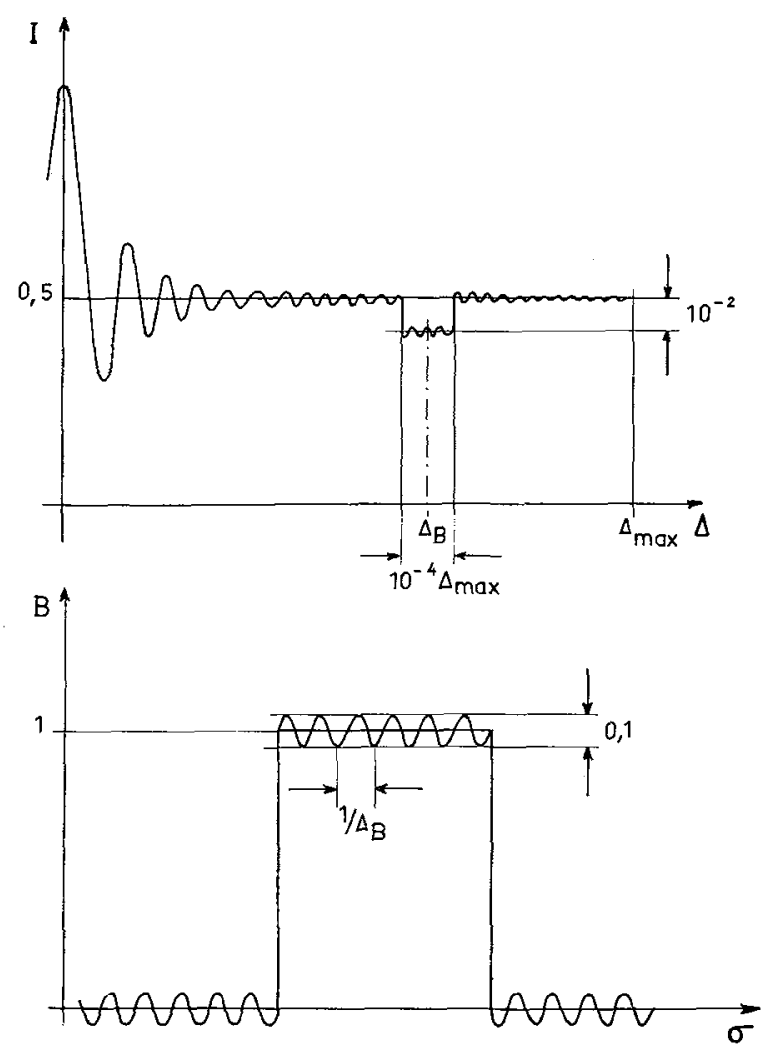

FIG. 2. - Interférogramme d'un spectre rectangulaire, perturbé par une brusque variation de niveau moyen et spectre correspondant. Figures non à l'échelle.

un spectre rectangulaire contenant $M=10^{4}$ éléments spectraux, étudié avec un pouvoir de résolution $R=10^{5}$ (valeurs correspondant effectivement à celles que nous avons atteintes sur Vénus vers $8000 \mathrm{~cm}^{-1}$ ). Supposons une baisse accidentelle de $1 \%$ de l'intensité moyenne de l'interférogramme pendant le défilement de 10 franges, c'est-à-dire $10^{-4}$ fois la durée totale d'enregistrement. Au spectre réel, se trouve alors superposée une ondulation parasite dont la hauteur 
pic à pic est égale à 0,1 , et qui pourrait facilement être prise pour une bande d'absorption.

Si cette baisse du signal était due à une fluctuation d'intensité de la source, elle serait facilement compensée par le dispositif d'intégration à temps variable. Mais si elle provient d'un petit déplacement du faisceau dans le système optique et en particulier sur les récepteurs, elle n'est pas connue et il n'est pas possible d'en tenir compte.

Un premier remède, indiqué par Fellgett [3] et que nous avons systématiquement employé lors de nos premiers essais [1] consiste à enregistrer un interférogramme équilibré (Fig. $3 a$ ) en prenant la différence

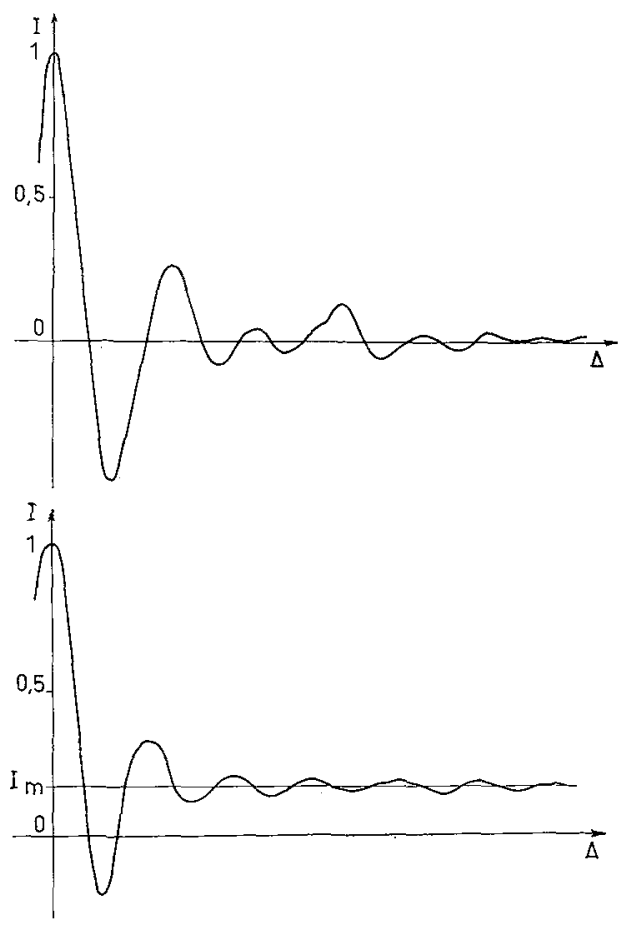

FIG. 3. - Interférogramme équilibré, de valeur moyenne nulle (en haut) et interférogramme imparfaitement équilibré (en bas).

entre les signaux donnés par deux récepteurs utilisant les deux faisceaux sortant de l'interféromètre et modulés en opposition. Cependant, ces deux faisceaux ne sont d'intensité égale que si le pouvoir réflecteur de la lame séparatrice est exactement $50 \%$ (et l'absorption nulle), ce qui n'est pas réalisable dans un domaine spectral étendu. En conséquence, le résultat obtenu est normalement celui de la figure $3 b$; il est pratiquement difficile d'obtenir pour $I_{m}$, intensité moyenne, une valeur inférieure à 0,1 .

D'autre part, cet équilibrage résulte d'une différence entre deux faisceaux ayant parcouru des trajets optiques en partie différents et tombant sur des récepteurs différents. Lorsque le faisceau est instable, l'équilibrage est Iui-même instable et les fluctuations observées de la valeur moyenne restent grandes même si $I_{m}$ est faible. Nos tentatives pour réduire artificiellement $I_{m}$ en atténuant par absorption l'un des faisceaux n'ont conduit à aucun résultat utile, les fluctuations restant les mêmes en valeur absolue.

Deux autres méthodes d'enregistrement destinées à réduire l'effet nuisible de la turbulence ont été indiquées, toutes les deux par Mertz. La première est la méthode de balayage rapide (《fast scan )), utilisée avec succès pour obtenir de nombreux spectres planétaires ou stellaires à basse résolution et décrite dans une communication à ce même Colloque [4].

Elle consiste à faire varier la différence de marche assez vite pour que les fréquences correspondant aux franges d'interférence qui défilent soient aussi élevées que le permet le récepteur utilisé (soit quelques centaines de $\mathrm{Hz}$ au plus pour une cellule au sulfure de plomb refroidie). Comme la turbulence atmosphérique possède un spectre de fréquences approximativement en $1 / f$, les composantes des fluctuations qui coïncident avec les fréquences Fourier sont fortement réduites. Le signal modulé par l'interféromètre est enregistré directement (sans modulation extérieure).

L'extension de cette méthode aux problèmes à haute résolution présente deux difficultés. D'une part, elle est incompatible avec l'enregistrement pas à pas qui présente de nombreux avantages et a seul, jusqu'ici, permis d'atteindre des pouvoirs de résolution élevés en laboratoire. D'autre part, la durée d'enregistrement est nécessairement très courte (par exemple, $50 \mathrm{~s}$ pour un pouvoir de résolution égal à $10^{4}$ si la fréquence moyenne des franges est de $200 \mathrm{~Hz}$ ). Or pour obtenir un rapport signal sur bruit suffisant sur des sources astronomiques faibles, il est indispensable d'utiliser pleinement le temps disponible qui est de l'ordre de quelques heures. Il est donc nécessaire de sommer un très grand nombre d'interférogrammes; ceci est facilement réalisable au moyen d'une mémoire digitale lorsque le nombre $N$ des échantillons n'est pas trop élevé (de l'ordre de 1000) mais devient difficile pour les grands nombres d'échantillons nécessaires pour nos enregistrements ( $N=58000$ jusqu'ici et vraisemblablement plus dans l'avenir lorsqu'un certain nombre de problèmes liés au temps de calcul auront été résolus).

La seconde méthode, décrite par Mertz lors du premier Colloque de Bellevue [5] mais non utilisée depuis, peut être appelée méthode de modulation interne et a été employée ici ; nous allons la discuter plus en détails. 
3. Méthode de modulation interne. - La méthode classique consiste à enregistrer l'interférogramme en cosinus

$$
I(\Delta)=\int_{0}^{\infty} B(\sigma) \cos 2 \pi \sigma \Delta \mathrm{d} \sigma=T F_{\cos }[B(\sigma)]
$$

par mesure de l'intensité $I\left(\Delta_{A}\right)$ en des points tels que $A$, d'abscisse $\Delta=\Delta_{A}$. Cette mesure est normalement effectuée en modulant le flux lumineux total par un modulateur extérieur à l'interféromètre.

Eliminons toute modulation externe et faisons osciller à une fréquence $N=\omega / 2 \pi$ une pièce de l'interféromètre (Fig. 4) de telle façon que la différence de mar-

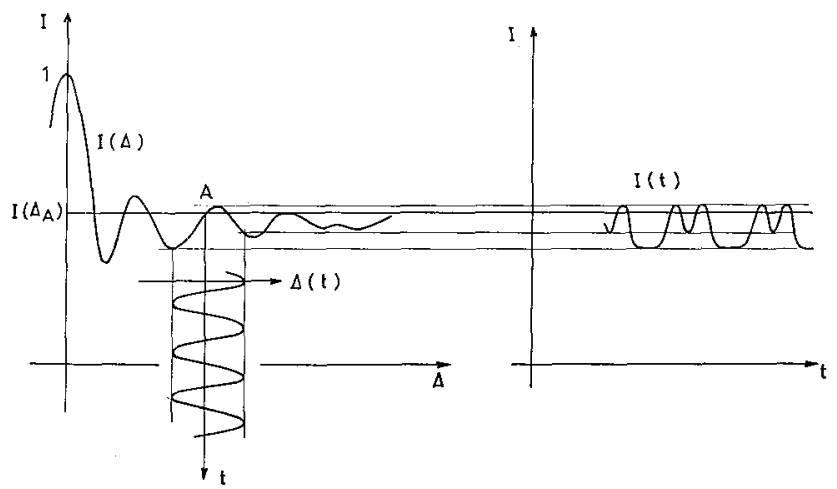

Fig. 4. - Modulation interne. La différence de marche varie suivant la loi $\Delta(t)$ au voisinage du point $A$, d'abscisse $\Delta_{\mathrm{A}}$; il y a exploration rapide d'une partie de la courbe $I(\Delta)$ et production d'un signal périodique $I(t)$.

che varie, par exemple, sinusoïdalement avec une amplitude. $\lambda_{0} / 4$ :

$$
\Delta=\Delta_{A}+\frac{\lambda_{0}}{4} \cos \omega t .
$$

L'intensité du signal sortant de l'interféromètre comprend un terme constant, qui ne sera pas utilisé, et des termes de fréquence $N, 2 N, 3 N, \ldots$ d'amplitudes $I_{1}, I_{2}, I_{3}, \ldots$

Posons $\sigma_{0}=1 / \lambda_{0}$. On montre que, au point $A$ :

$$
\begin{aligned}
& I_{1}\left(\Delta_{A}\right)=\int_{0}^{\infty} 2 J_{1}\left(\frac{\pi}{2} \frac{\sigma}{\sigma_{0}}\right) B(\sigma) \sin 2 \pi \sigma \Delta_{A} \mathrm{~d} \sigma \\
& I_{2}\left(\Delta_{A}\right)=\int_{0}^{\infty} 2 J_{2}\left(\frac{\pi}{2} \frac{\sigma}{\sigma_{0}}\right) B(\sigma) \cos 2 \pi \sigma \Delta_{A} \mathrm{~d} \sigma \\
& I_{3}\left(\Delta_{A}\right)=\int_{0}^{\infty} 2 J_{3}\left(\frac{\pi}{2} \frac{\sigma}{\sigma_{0}}\right) B(\sigma) \sin 2 \pi \sigma \Delta_{A} \mathrm{~d} \sigma,
\end{aligned}
$$

e c... où $J_{1}, J_{2}, J_{3}, \ldots$ sont les fonctions de Bessel d'ordre $1,2,3, \ldots$ En opérant sur le signal des démodulations synchrones aux fréquences $N, 2 N, 3 N, \ldots$ il est possible de mesurer les amplitudes des différents harmoniques pour toutes les valeurs utiles de $\Delta_{A}$ et par conséquent d'enregistrer les interférogrammes :

$$
\begin{aligned}
& I_{1}(\Delta)=T F_{\sin }\left[2 J_{1}\left(\frac{\pi}{2} \frac{\sigma}{\sigma_{0}}\right) B(\sigma)\right] \\
& I_{2}(\Delta)=T F_{\cos }\left[2 J_{2}\left(\frac{\pi}{2} \frac{\sigma}{\sigma_{0}}\right) B(\sigma)\right] \\
& I_{3}(\Delta)=T F_{\sin }\left[2 J_{3}\left(\frac{\pi}{2} \frac{\sigma}{\sigma_{0}}\right) B(\sigma)\right], \text { etc. }
\end{aligned}
$$

En calculant alors leurs transformées de Fourier, en cosinus pour les termes pairs ou en sinus pour les termes impairs nous obtiendrons le spectre $B(\sigma)$ mais multiplié par l'une des fonctions $J_{1}, J_{2}, \ldots$ (Fig. 5).

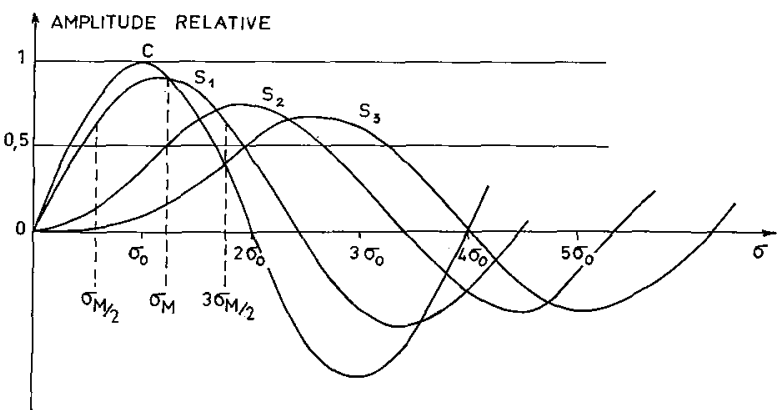

Fig. 5. - Facteur par lequel est multiplié le spectre $B(\sigma)$ dans le cas d'une modulation rectangulaire $(C)$, ou d'une modulation sinusoïdale $\left(S_{1}, S_{2}, S_{3}\right.$ correspondant aux trois premiers harmoniques).

Chacun de ces spectres n'est donc utilisable que dans un domaine limité - c'est la principale restriction apportée par la méthode — et le rendement $\left({ }^{7}\right)$ est partout inférieur à l'unité.

Cependant, ce rendement reste acceptable. On montre que, en détectant seulement le premier harmonique le rendement maximum est 0,91 pour $\sigma_{M}=1,18 \sigma_{0}$ et qu'il reste supérieur à 0,64 entre $\sigma_{M} / 2$ et $3 \sigma_{M} / 2$, c'est-à-dire dans un domaine assez large pour correspondre à celui de la cellule au sulfure de plomb (3 000 à $9000 \mathrm{~cm}^{-1}$ environ).

(7) Comparé à celui donné par une méthode de modulation externe optimum, c'est-à-dire une modulation rectangulaire avec modulateur réfléchissant, du type de celle que nous avons employée jusqu'ici (1). Dans les deux cas, les deux sorties de l'interféromètre sont supposées utilisées, ce qui nécessite deux récepteurs. 
Remarquons enfin qu'une modulation rectangulaire de même amplitude conduit à un signal modulé dont les harmoniques ont pour amplitude :

$$
\begin{aligned}
& I_{1}^{\prime}(\Delta)=T F_{\sin }\left[\frac{4}{\pi} \sin \left(\frac{\pi}{2} \frac{\sigma}{\sigma_{0}}\right) B(\sigma)\right] \\
& I_{3}^{\prime}(\Delta)=T F_{\sin }\left[\frac{4}{3} \frac{4}{\pi} \sin \left(\frac{\pi}{2} \frac{\sigma}{\sigma_{0}}\right) B(\sigma)\right],
\end{aligned}
$$

etc...

Seul le terme $I_{1}^{\prime}$ est intéressant, les suivants ne permettant pas de couvrir un domaine spectral différent. Le rendement maximum est maintenant égal à l'unité pour $\sigma=\sigma_{0}$ et reste supérieur à 0,71 pour

$$
\sigma_{0} / 2<\sigma<3 \sigma_{0} / 2 \text {. }
$$

Ce type de modulation est donc légèrement préférable à la modulation sinusoïdale (mais de réalisation plus difficile).

L'avantage essentiel de la modulation interne, comparée à l'enregistrement direct des intensités est celui indiqué par Mertz [5] : le niveau moyen de l'interférogramme est rigoureusement nul, quelles que soient les fluctuations de la source. Ce résultat reste vrai pour de petits déplacements des faisceaux; en effet, il n'est pas nécessaire pour l'obtenir d'équilibrer les signaux de deux récepteurs différents. Il nous a donc paru utile de chercher à combiner cette propriété essentielle pour les observations à travers l'atmosphère et celles de l'enregistrement pas à pas.

4. Réalisation. - 1) PARTIE OPTIQUE. L'interféromètre a déjà été décrit [1]. Il est traversé par deux faisceaux différents géométriquement séparés : l'un est le faisceau infrarouge à mesurer et l'autre un faisceau de lumière verte du mercure utilisé pour le comptage et l'asservissement. Il a été employé ici sans autre modification que l'adjonction de 4 lames planes et parallèles de fluorine placées sur le faisceau infrarouge seul. Le faisceau de contrôle n'est donc pas affecté et le déplacement pas à pas du chariot reste conforme à la description déjà donnée.

La normale aux lames est inclinée d'environ $10^{\circ} \mathrm{par}$ rapport à la direction du faisceau. Chaque paire produit une translation nulle des rayons ; il n'y a donc pas décentrement des anneaux à l'infini donnés par l'interféromètre.

Une paire de lames est portée par les deux branches d'un diapason autoentretenu (Fig. 6) ; l'amplitude de l'oscillation - de l'ordre de quelques microns - est réglable. L'autre paire de lames, placée sur l'autre bras, sert seulement à conserver l'achromatisme de l'interféromètre ; l'une des lames possède un réglage fin d'incli-

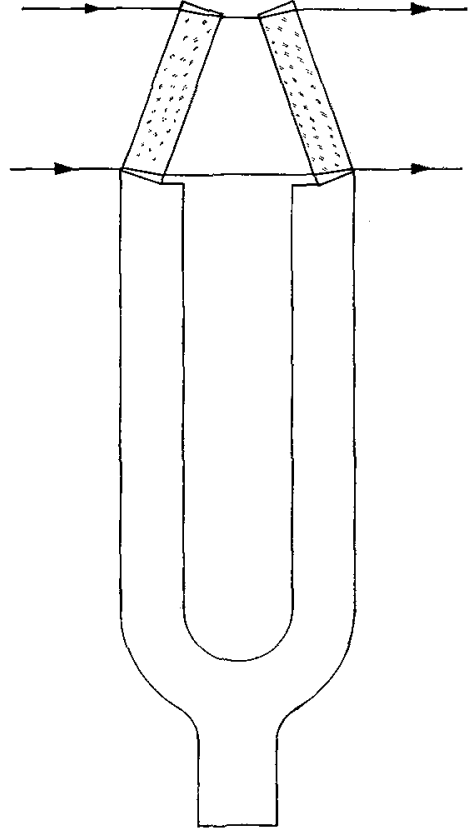

FIG. 6. - Paire de lames vibrantes en fluorine placée sur le trajet du faisceau infrarouge dans l'interféromètre.

naison. Les lames sont planes, parallèles et d'épaisseurs égales à environ $1 / 2$ frange (visible) près.

Pour que la loi de variation $\Delta(t)$ cherchée soit obtenue, les lames vibrantes ne doivent introduire ni dérive de la différence de marche ni réaction oscillatoire sur le bâti de l'interféromètre. En effet, l'asservissement ne pourrait compenser ni une variation de l'inclinaison moyenne de l'une des lames - puisque le faisceau de contrôle ne les traverse pas - ni une légère oscillation du chariot mobile induite par couplage mécanique car la fréquence choisie $(200 \mathrm{~Hz})$ est trop élevée pour son temps de réponse $(5 \mathrm{~ms})$.

Bien qu'un diapason correctement équilibré n'exerce qu'une réaction minime sur son support, il a été nécessaire de le placer sur un bâti entièrement indépendant de celui de l'interféromètre. Dans ces conditions, de petites rotations relatives du support du diapason et de celui de l'interféromètre sont à craindre, mais la variation correspondante de la différence de marche est du second ordre, car le diapason se comporte alors comme une lame plane et parallèle unique, perpendiculaire au faisceau.

Enfin, l'amplitude de l'oscillation doit être stable. En effet, une variation de cette amplitude produit une affinité de la courbe $J_{1}(\sigma)$ par laquelle est multiplié le spectre, et une variation des intensités calculées. L'effet peut être faible si le domaine spectral est étroit et voisin du maximum de $J_{1}$. 


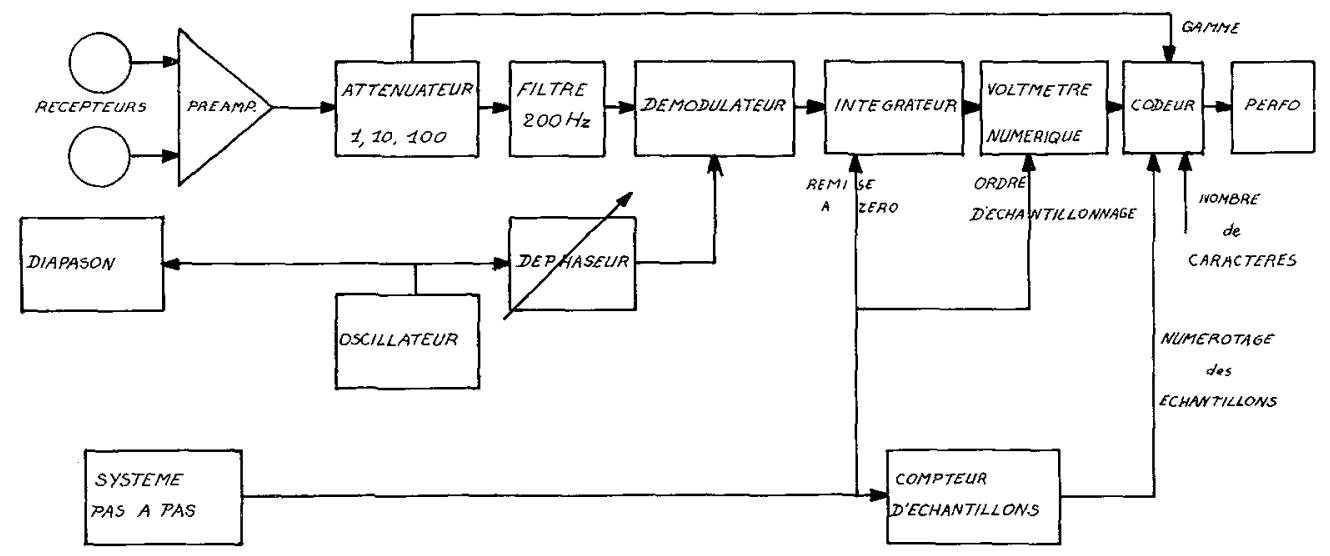

FIG. 7. - Schéma de principe du système d'enregistrement (partie analogique et partie digitale).

Un dispositif de stabilisation électronique de l'amplitude du diapason prévu à l'origine n'a pu être réalisé à temps pour les expériences, et l'amplitude de l'oscillation, bien que suffisamment stable au cours d'un enregistrement, n'était pas reproductible avec assez de précision d'un jour à l'autre. En conséquence, la reproductibilité des intensités dans les spectres calculés a été jusqu'ici un peu moins bonne $\left({ }^{8}\right)$ qu'avec la modulation externe utilisée auparavant [1].

2) Partie électrique (Fig. 7). Les deux récepteurs utilisant les deux sorties de l'interféromètre fournissent deux signaux en opposition; leur différence est amplifiée par un préamplificateur à large bande. Le signal traverse ensuite un atténuateur à commande manuelle donnant des atténuations égales à $1,10 \mathrm{ou}$ 100 , puis un filtre centré sur la fréquence de modulation $(200 \mathrm{~Hz})$ et de largeur de bande $40 \mathrm{~Hz}$ environ; il est ensuite démodulé par un relais vibrant (choisi en raison de sa parfaite linéarité et de son absence de dérive) puis intégré.

Seul le premier harmonique de fréquence $N$ doit être mesuré ; il est nécessaire que les harmoniques pairs en particulier soient aussi fortement atténués que possible : en effet, ils donneraient des interférogrammes $I_{2}, I_{4}, \ldots$ en quadrature avec l'interférogramme désiré $I_{1}$, ce qui se traduirait par une erreur de phase.

(8) Ce dispositif de modulation interne de réalisation relativement simple et rapide n'est pas considéré comme la solution définitive. De meilleurs résultats devraient pouvoir être obtenus sans l'adjonction de pièces optiques supplémentaires en faisant osciller l'un des miroirs de l'interféromètre - par exemple le petit miroir de l'un des " ceils de chat»-qui serait porté par une céramique piezo électrique. L'asservissement pourrait alors contrôler à la fois la valeur moyenne de la différence de marche et l'amplitude de l'oscillation; une forme d'oscillation rectangulaire serait réalisable.
Le filtre donne une première atténuation (insuffisante par elle-même) des signaux de fréquence $2 N, 3 N, \ldots$; il facilite en même temps le réglage de phase de la démodulation en donnant au signal transmis une allure sinusoïdale. La démodulation elle-même fournit une atténuation plus grande $\left({ }^{9}\right)$, de l'ordre de $2 N T$ pour l'harmonique 2 , où $T$ est le temps d'intégration (soit environ 100 fois avec $N=200 \mathrm{~Hz}$ et $T=0,25 \mathrm{~s}$ ). Cette atténuation pourrait être rendue infinie en prenant pour temps d'intégration un multiple entier de la période $1 / 2 N$ de l'harmonique 2 -le plus gênant. Cette précaution n'a pas été jugée nécessaire jusqu'ici mais le deviendrait pour des temps d'intégration sensiblement plus courts.

A la différence de la modulation externe précédemment utilisée, la modulation interne ne permet pas d'obtenir un signal proportionnel à l'intensité totale du flux reçu, en vue de régler le temps d'intégration. Dans le cas de Jupiter (observations de nuit), le temps d'intégration a été réglé à partir du signal donné par les photomultiplicateurs du système de guidage [1]; ce procédé est évidemment très grossier, les photomultiplicateurs mesurant l'intensité visible. Les résultats ont néanmoins été assez satisfaisants : des spectres reproductibles au bruit près ont été obtenus soit par ciel clair, soit par ciel très absorbant et même avec absorption rapidement fluctuante (cirrus).

Le cas de Vénus (observation de jour) est plus difficile : l'effet le plus gênant de nuages légers est alors non pas l'atténuation du signal utile mais la production d'un signal parasite non négligeable; dans ces

(9) A condition que dans le fonctionnement du relais les deux demi-périodes soient rigoureusement égales; le réglage est contrôlé en appliquant à l'entrée du démodulateur une tension continue et en vérifiant que la sortie de l'intégrateur reste nulle. 
conditions, nous n'avons pas essayé jusqu'ici de faire varier le temps d'intégration.

La solution à ces difficultés (non encore expérimentée) paraît être l'emploi simultané d'une modulation interne à une fréquence $N$ et d'une modulation externe à fréquence $N^{\prime}$ par miroir vibrant, modulant sélectivement le flux de la planète et non celui du fond du ciel, ni l'émission thermique des miroirs. On obtiendrait alors, par détection synchrone à la fréquence $N^{\prime}$ un signal proportionnel à l'intensité totale de la planète dans la fenêtre infrarouge étudiée, qui permettrait de régler plus correctement le temps d'intégration. D'autre part, deux détections synchrones en cascade aux fréquences $N$ et $N^{\prime}$ fourniraient un interférogramme débarrassé des composantes parasites (fond du ciel et émission thermique). Ce système d'enregistrement deviendra sans doute indispensable dans le cas d'astres plus faibles, ou pour l'étude de longueurs d'onde plus grandes (fenêtre 8-12 $\mu$ en particulier).

\section{Quantification et enregistrement de l'interféro-} gramme. - Le nombre élevé des points mesurés sur l'interférogramme (jusqu'à 42000 sur Vénus et 58000 sur des essais en laboratoire) impose de chercher à réduire le plus possible le volume des données à enregistrer sur la bande perforée, spécialement en vue de réduire le temps de transmission à l'ordinateur par la ligne téléphonique - dont le débit est limité.

D'autre part, le nombre des éléments spectraux (jusqu'à 20000 sur Vénus) et par conséquent le rapport élevé entre l'intensité maximum et le bruit dans l'interférogramme obligent à accroître le plus possible la dynamique $\mathrm{du}$ système d'enregistrement : le signal maximum doit en effet rester inférieur à celui pour lequel un défaut de linéarité non négligeable de la partie analogique (préamplificateur et intégrateur) apparaitrait; par ailleurs, la valeur efficace du bruit doit rester nettement supérieure au signal minimum mesurable, fixé par la dérive de l'intégrateur d'une part, et le niveau de quantification d'autre part. Ces points ont déjà été brièvement discutés [1]; il a été montré qu'un facteur $q=M S_{\text {moy }} / B_{\text {eff }}$ produit du nombre d'éléments spectraux $M$ par le rapport du signal moyen $S_{\text {moy }}$ à la valeur efficace du bruit $B_{\text {eff }}$ dans le spectre définit la qualité du système complet à ce point de vue : toute erreur systématique ou aléatoire dans la mesure de l'intensité de l'interférogramme entraîne une réduction de $q$; ce facteur mesure en quelque sorte la perfection du multiplexage obtenu.

Ces deux difficultés (réduction du volume des données et extension de la dynamique) ont été résolues de la façon suivante (Fig. 8) : au début de l'enregistrement, le signal de l'intégrateur est mesuré par un volt-

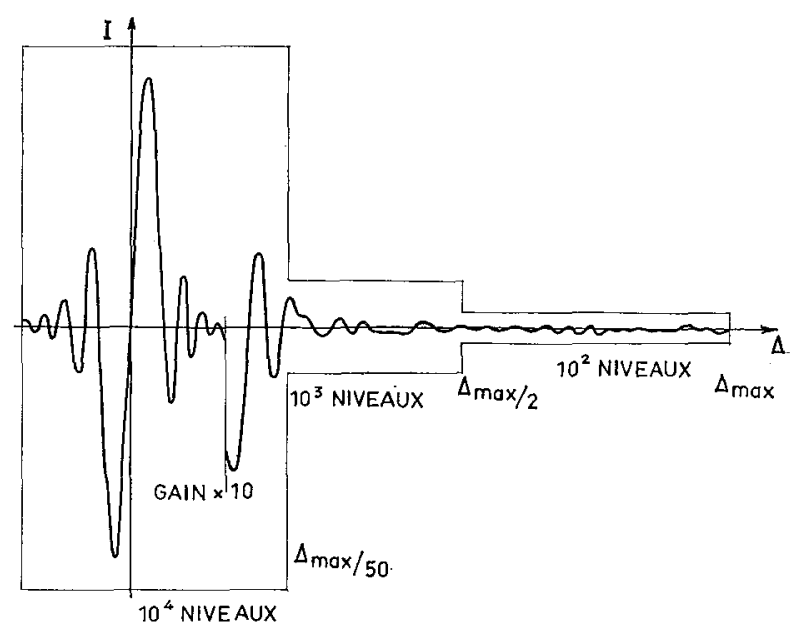

FIG. 8. - Quantification de l'interférogramme. Les valeurs. de la différence de marche $\Delta$ pour lesquelles interviennent l'augmentation du gain et la réduction du nombre de niveaux sont très variables suivant les cas. La figure n'est pas à l'échelle.

mètre numérique donnant 4 chiffres significatifs; la portion centrale de l'interférogramme est donc quantifiée à $10^{4}$ niveaux. Les indications du voltmètre sont codées en code $\mathrm{BCD}$, qui nécessite un caractère par chiffre décimal ; chaque mesure est donc transcrite par 4 caractères sur une bande perforée.

Lorsque le signal modulé a suffisamment décru, on le multiplie par 10 au moyen de l'atténuateur; le bruit du récepteur est multiplié par le même facteur et devient supérieur au niveau de quantification $\left({ }^{10}\right)$.

L'opération est pratiquement équivalente à une multiplication par 10 de la dynamique de l'intégrateur. L'emploi de 5 chiffres significatifs coûterait un caractère supplémentaire et ne donnerait pas de gain effectif de précision; celle-ci serait alors limitée par la linéarité et la stabilité de la partie analogique. Le signal continuant à décroître, les décades de poids supérieur du voltmètre deviennent successivement nulles; on peut réduire le nombre de caractères à 3 puis à 2 , c'està-dire le nombre de niveaux à $10^{3}$ et $10^{2}$ sans perte de précision. Suivant la structure de l'interférogramme, cette réduction intervient plus ou moins tôt : la figure 8 donne des ordres de grandeur.

Les mesures sont groupées par blocs de 10 qui sont numérotés grâce à un compteur déclenché par l'avance de l'interféromètre. Au début de chaque bloc, sont perforés 8 caractères supplémentaires indiquant le

(10) Un deuxième changement de gain d'un facteur 10 peut être employé ; il est utile pour les spectres d'absorption en laboratoire, pour lesquels le rapport $s / b$ peut être beaucoup plus élevé que pour les spectres planétaires, ou pour des spectres solaires. 


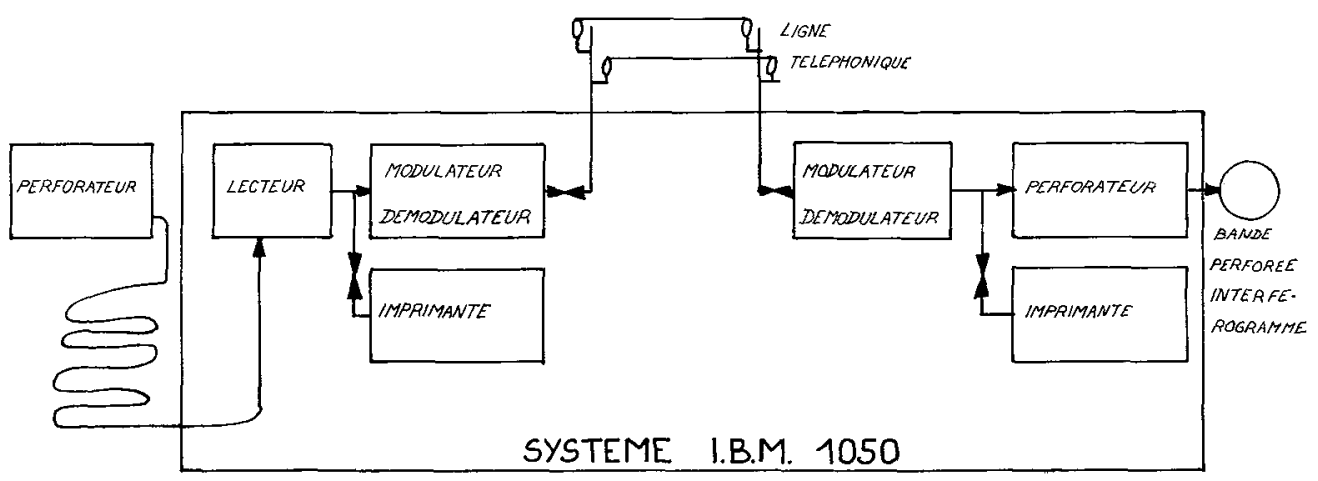

SAINT MICHEL

MEUDON

FIG. 9. - Transmission de l'interférogramme de l'Observatoire de Saint-Michel au Centre de Calcul Numérique de Meudon.

numéro du bloc ( 4 caractères permettant de repérer $10^{4}$ blocs, soit $10^{5}$ mesures), la gamme de l'atténuateur, le nombre de caractères par mesure et 2 caractères spéciaux nécessités par les contrôles pendant la transmission. Le nombre moyen de caractères par mesure est ainsi égal à 4,8 au début de l'interférogramme puis passe à 3,8 et 2,8 .

De cette façon, le nombre total de caractères n'a pas dépassé 136000 pour un enregistrement de 42000 mesures (soit en moyenne 3,2 caractères/mesure) alors que sans ces artifices, pour la même précision, il aurait atteint 240000 . Comme il sera montré plus loin, cette réduction était indispensable.

6. Transmission des données et calcul du spectre. Afin de faire le plus rapidement possible les vérifications indispensables sur le spectre, la bande perforée est aussitôt transmise à Meudon en une opération continue, sans stockage intermédiaire (Fig. 9), grâce à une ligne téléphonique ordinaire. Le système $1050 \mathrm{IBM}$ qui permet de transmettre 10 caractères/s est utilisé et reproduit à Meudon une bande perforée identique. De plus, deux imprimantes, à chaque bout de la ligne, donnent en clair la liste des valeurs de l'interférogramme.

La durée maximum normale $\left({ }^{1}{ }^{1}\right)$ d'un enregistrement

(11) C'est-à-dire, en l'absence de nuages; en pratique, dans de nombreux cas, les interruptions par les nuages ont été fréquentes et la durée des enregistrements s'est trouvée augmentée. La durée de 3 heures correspond pratiquement à la cadence maximum possible avec le système actuel : pour mesurer 42000 points, il faut faire $4 \mathrm{mesures} / \mathrm{s}$, c'est-à-dire que l'on dispose de $250 \mathrm{~ms}$ par point de l'interférogramme. Environ $70 \mathrm{~ms}$ étant nécessaires pour le déplacement du chariot de l'interféromètre, la remise à zéro de l'intégrateur et la mesure par le voltmètre, le temps utile restant pour l'intégration est seulement $180 \mathrm{~ms}$. Une cadence plus rapide gaspillerait une fraction excessive du temps d'enregistrement. Les valeurs données ici correspondent au cas de Vénus - le plus difficile. Pour Jupiter, on avait $N_{\max }=13000$ et la cadence était donc plus lente. a été prise égale à 3 heures, ce qui permet de faire en une journée, 3 enregistrements planétaires, dont l'un au voisinage du méridien et les deux autres à des élévations plus basses, et un enregistrement de comparaison avec le soleil. La durée totale d'enregistrement effectif est alors de 12 heures, réparties sur 13 ou 14 heures environ, en raison des contrôles divers au début de chaque enregistrement, et des changements nécessaires pour passer de la planète au soleil.

La durée de transmission dans le cas d'un enregistrement de 136000 caractères est alors de $3 \mathrm{~h} 45 \mathrm{mn}$, soit environ $15 \mathrm{~h}$ par journée de 4 interférogrammes, et la transmission a tendance à prendre du retard par rapport aux enregistrements. La situation que nous venons de décrire est d'ailleurs quelque peu théorique, les nuages venant à tout instant ralentir les enregistrements, et les incidents sur la ligne téléphonique, les erreurs de transmission et les messages indispensables ralentissant la transmission. La bande perforée joue le rôle nécessaire de volant et permet de donner une indépendance suffisante aux deux opérations.

Après la fin de chaque transmission, la bande perforée transmise est lue à Meudon par un lecteur IBM 1011 et les données décodées et transférées sur bande magnétique par un ordinateur 1401 (périphérique de l'ordinateur central 7040) (Fig. 10). La rapidité relative de cette lecture ( 500 caractères/s, durée maximum : 5 minutes) rend inutile de l'effectuer pendant la transmission elle-même. Au cours de cette opération, la bande est vérifiée et des corrections peuvent être introduites au moyen de cartes perforées manuellement pour éliminer les erreurs de perforation (à St-Michel ou à Meudon) et de transmission. Comme ces corrections exigent de transmettre des messages (avec les imprimantes) elles font souvent perdre beaucoup de temps. 

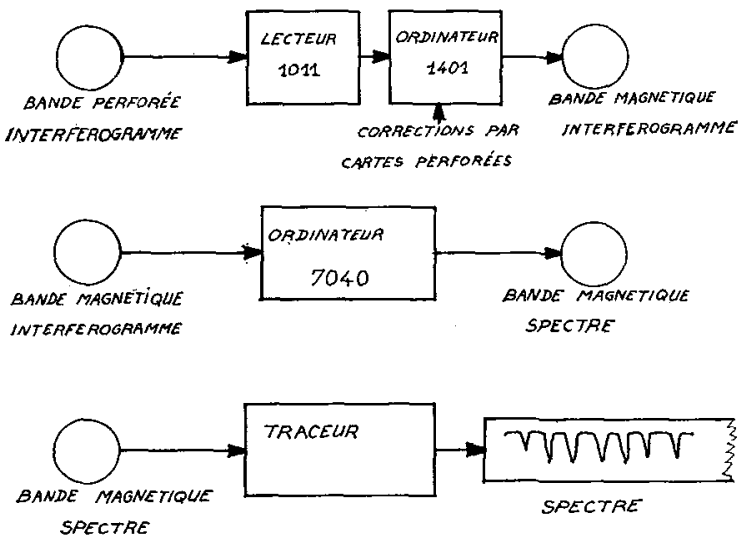

FIG. 10. - Lecture de la bande, calcul et tracé du spectre.

Le calcul de 3 étroites tranches du spectre (contenant environ 100 éléments spectraux chacune) est alors effectué par l'ordinateur central. La position des 3 tranches est choisie à l'avance : l'une est prise au voisinage du maximum de transmission du filtre dans une région où une (ou plusieurs) raie d'absorption fine et isolée est attendue, les deux autres de part et d'autre de la bande passante du filtre. On obtient ainsi les renseignements indispensables : rapport signal/bruit, et résolution effective. Le temps de lecture du programme et les données (toujours pour $N=42000$ ) est de 15 minutes, et le temps de calcul des 3 échantillons est de 60 minutes $\left({ }^{12}\right)$.

Les résultats sont alors présentés sous forme de courbe par un traceur digital rapide (Benson-France, $300 \mathrm{pas} / \mathrm{s}$ ) et les résultats communiqués à l'expérimentateur 80 à 90 minutes après la fin de la transmission. Les spectres reconnus bons sont calculés ultérieurement dans toute leur étendue.

$\mathrm{On}$ voit que dans ces conditions, les résultats de l'interférogramme $n$ sont connus à temps pour corriger (éventuellement) le mode opératoire pour l'interférogramme $n+2$ mais non pas pour l'interférogramme $n+1$. Une réduction des temps de transmission et de calcul est donc très souhaitable $\left({ }^{13}\right)$.

(12) Durant les expériences, le seul programme disponible était encore celui - déjà décrit [6] - qui effectue la transformée de Fourier point par point, et pour lequel le temps de calcul obéit à la formule $T=M N \times 3 \times 10^{-4} \mathrm{~s}$ avec un ordinateur 7040. Après la fin des expériences, un nouveau programme utilisant la méthode de Cooley et Tukey [7] et de Forman [8] est devenu utilisable; il a permis de ramener le temps de calcul à 5 heures jusqu'à $M=N=58000$ et a été employé pour le calcul des spectres complets.

(13) Cependant, dans quelques cas, nous avons aussi effectué le calcul du spectre - à résolution réduite - à partir de la fraction d'interférogramme déjà transmise pendant que l'enregistrement de celui-ci se poursuivait. Le calcul était alors beaucoup plus rapide et le résultat obtenu avant la fin de l'enregistrement.
La réduction du temps de transmission peut être obtenue de deux façons différentes. D'une part, la capacité du système de transmission a jusqu'iciété mal employée ; il permet en effet de reproduire 64 caractères différents, donc de transmettre 6 bits par caractère. Or, seuls les 10 caractères numériques étaient effectivement utilisés, ce qui donnait $\log _{2} 10=3,32$ bits par caractère. L'emploi d'un codeur binaire permettrait de transmettre $6 / 3,32=1,80$ fois plus d'information avec le même nombre de caractères; la longueur de la bande perforée et le nombre moyen d'erreurs seraient réduits dans la même proportion.

De plus, des équipements plus rapides, transmettant jusqu'à 150 caractères par seconde sont maintenant disponibles. Il est donc possible de réduire le temps de transmission par un facteur de l'ordre de 30 par rapport à nos expériences; dans ces conditions, il ne serait plus indispensable de disposer d'une ligne téléphonique louée en permanence. La transmission ne durerait plus que quelques minutes et pourrait se faire sur le réseau commuté.

Il est nécessaire d'autre part, de réduire non seulement le temps de calcul lui-même mais aussi celui pris par les diverses manipulations de bandes à l'arrivée des données. C'est précisément ce que permettent les ordinateurs de la nouvelle génération auxquels plusieurs utilisateurs peuvent avoir simultanément un accès direct (time sharing).

Dans ces conditions, et compte tenu des nouveaux programmes, il est raisonnable de penser que le calcul à la résolution maximum d'une tranche d'un spectre assez large pour vérifier sa qualité quelques minutes après la fin de l'enregistrement sera d'ici quelques temps une opération courante. II sera également possible, sans que cela pose aucun problème supplémentaire, de généraliser le calcul (à résolution réduite) d'une tranche spectrale à partir de la fraction d'interférogramme déjà enregistrée, ce calcul pouvant même être effectué à plusieurs reprises. Ce résultat sera aussi commode que celui donné par un calcul en temps réel, et ceci sans que l'ordinateur ou la ligne de transmission soient bloqués en permanence.

7. Résultats. - 1) RÉDUCTION DES EFFETS DE LA TURBULENCE. La figure 11 illustre l'effet perturbateur de la turbulence tel qu'il se présentait avec le dispositif de modulation extérieure précédemment utilisé [1]. Elle représente un enregistrement du signal infrarouge obtenu après démodulation et filtrage, l'interféromètre étant maintenu stationnaire en une position de différence de marche élevée telle que l'interférogramme coïncide pratiquement avec sa valeur 


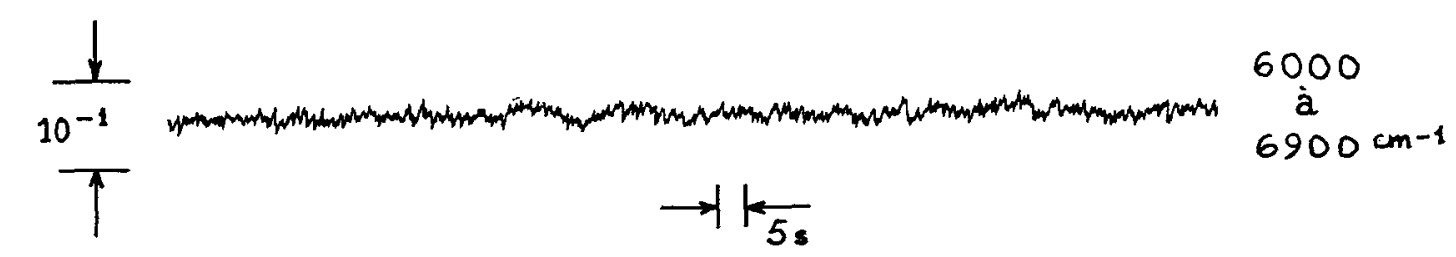

FIG. 11. - Bruit de turbulence, modulation externe, faisceau de Mars, rapporté à l'amplitude de l'interférogramme au centre. Constante de temps $T=0,4 \mathrm{~s}$.

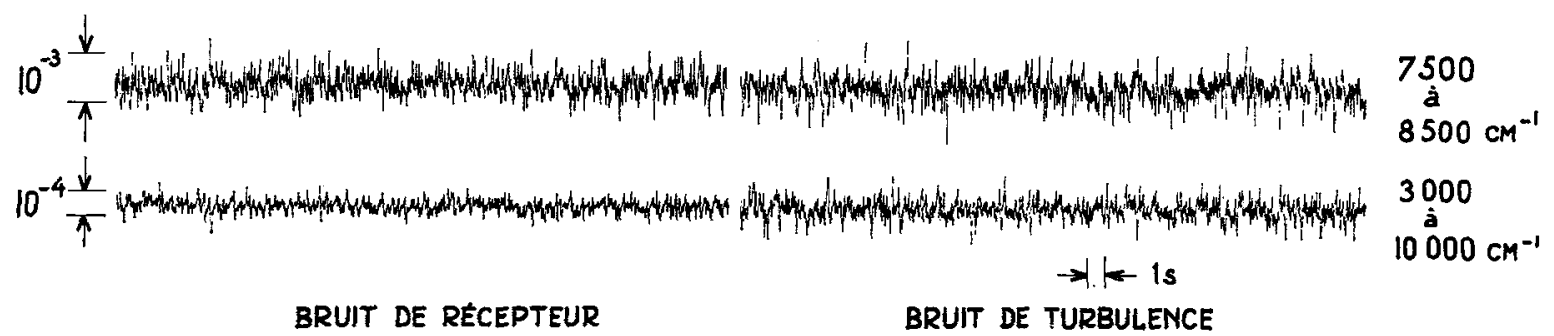

FIG. 12. - Bruit de récepteur seul (cellưles dans l'obscurité), et bruit de turbulence avec modulation interne. Faisceau de Vénus. Constante de temps $0,1 \mathrm{~s}$.

moyenne. On notera en particulier la présence dans cet enregistrement du (bruit de turbulence», de composantes basses fréquences très supérieures à celles du bruit de récepteur et dont l'amplitude est de l'ordre de 1 à $2 \%$ de celle de la portion centrale de l'interférogramme. Le signal provenait de Mars, à travers un filtre isolant sensiblement la fenêtre à $1,6 \mu$ (observation de nuit, hauteur au-dessus de l'horizon : $h=52^{\circ}$ ).

La figure 12 montre le progrès considérable donné par la modulation interne malgré des conditions de turbulence beaucoup plus sévères (Vénus, observation de jour : $h=32^{\circ}$ ). L'accroissement par rapport au pur bruit de récepteur est négligeable dans le cas d'un domaine spectral restreint, et appréciable seulement lorsque la totalité du domaine accessible aux récepteurs est utilisée.

2) JUPITER. La figure 13 donne à titre de comparaison les meilleurs spectres de Jupiter obtenus jusqu'ici dans le proche infrarouge $(1 \mu$ à $1,65 \mu)$ par des procédés classiques par Kuiper [9] et par Moroz [10]. De même, la figure 14 reproduit un spectre obtenu par Delbouille, Roland et Gebbie [11] par transformation de Fourier. Pour permettre d'évaluer la résolution (qui est peu différente dans les 3 cas), nous donnons dans la figure 15 un spectre obtenu par transformation d'un de nos interférogrammes (enregistré sans filtre afin de couvrir un domaine étendu) dont la longueur a été réduite, lors du calcul, jusqu'à ce que son aspect reproduise approximativement celui des spectres précédents. La limite de résolution

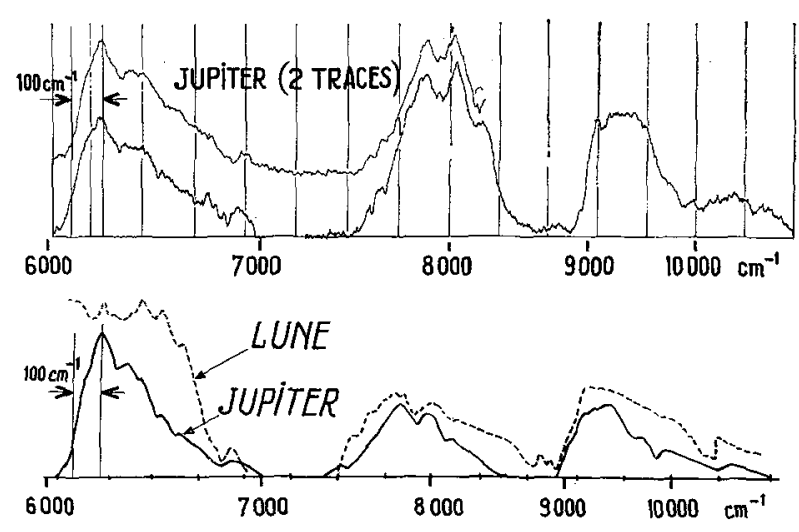

FIG. 13. - Spectres de Jupiter dans le proche infrarouge obtenus avec des spectromètres à réseau :

En haut, Kuiper ([9], Fig. 12 et 13), Observatoire de Mac Donald, télescope de $205 \mathrm{~cm}, 2$ spectres de Jupiter.

En bas, Moroz ([10], Fig. 9), Observatoire de Crimée, télescope de $125 \mathrm{~cm}$. Un spectre de la lune et un spectre de Jupiter (moyenne de 3 spectres indépendants) sont présentés. Temps d'observation total pour Jupiter : 50 minutes. Dans les 2 cas, nous avons inversé les figures de droite à gauche et ajouté une échelle en $\mathrm{cm}^{-1}$ pour rendre les spectres comparables à ceux des figures 14 et 15.

ainsi trouvée est égale à $30 \mathrm{~cm}^{-1}$. L'accord entre les 4 courbes est raisonnable; mais comme il s'agit d'un domaine spectral large, il faudrait tenir compte de facteurs tels que l'angle de blaze des réseaux, le rendement des lames séparatrices et (dans notre cas) du facteur $J_{1}\left(\pi / 2 \sigma / \sigma_{0}\right)$ avec $\sigma_{0} \simeq 6500 \mathrm{~cm}^{-1}$. 


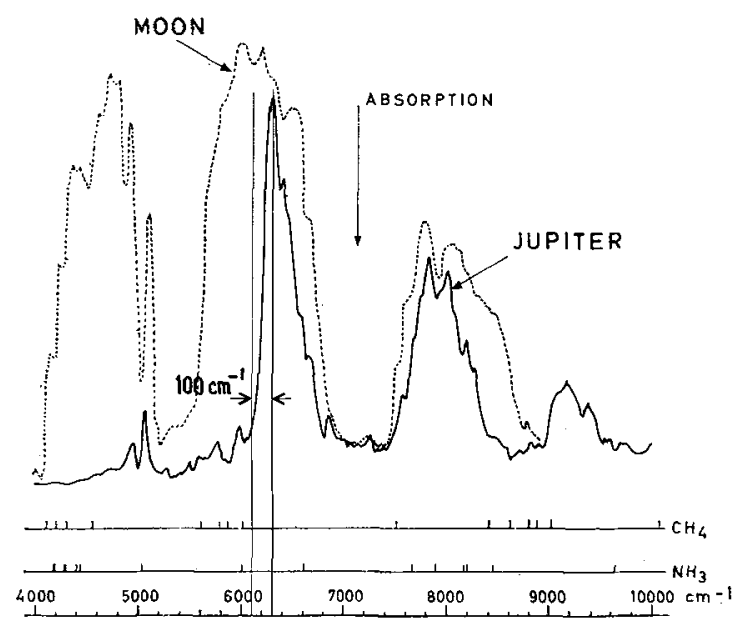

Frg. 14. - Spectre de Jupiter couvrant une région un peu plus étendue obtenu par spectroscopie de Fourier par Delbouille et al. ([11], Fig. 1). Observatoire de Lick, télescope de $3 \mathrm{~m}$. Temps d'observation : 8 minutes.

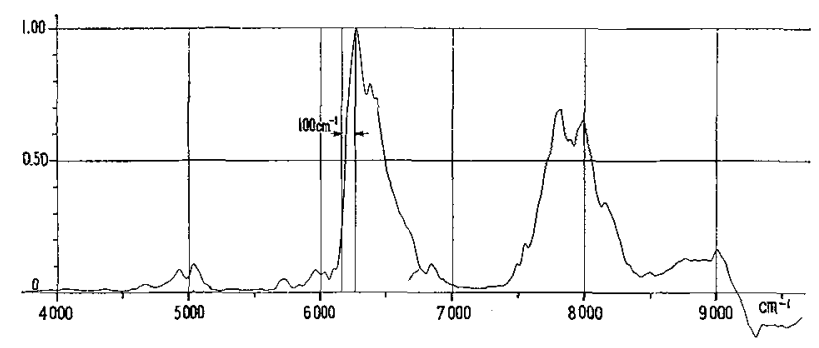

Fig. 15. - Spectre de Jupiter obtenu par réduction de la longueur d'un de nos interférogrammes, enregistré sans filtre avec un pas égal à 1 frange de référence $\left(\sigma_{\mathrm{r}}=18300 \mathrm{~cm}^{-1}\right)$. Résolution $\delta \sigma=30 \mathrm{~cm}^{-1}$ sensiblement égale à celle de Kuiper, comme le montre la bande de $\mathrm{O}^{2}$ (tellurique) à $7900 \mathrm{~cm}^{-1}$ et légèrement meilleure que celle des deux autres courbes. Au-delà du point $\sigma=\sigma_{\mathrm{r}} / 2=9150 \mathrm{~cm}^{-1}$, le spectre se répète identique à lui-même; entre 8500 et $9150 \mathrm{~cm}^{-1}$, les intensités sont perturbées (phénomène de recouvrement d'ordre) par celles de la région $9150-9800 \mathrm{~cm}^{-1}$. Temps d'observation : 5 minutes. Observatoire de Saint-Michel, télescope de $193 \mathrm{~cm}$.

De plus, dispersion et résolution sont variables sur la figure 12 et constantes sur les figures 14 et 15 .

La figure 16 montre deux spectres de Jupiter présentant la meilleure résolution que nous ayons atteinte jusqu'ici $\left(0,3 \mathrm{~cm}^{-1}\right)$. La portion représentée a une largeur de $100 \mathrm{~cm}^{-1}$, soit environ $1 / 10 \mathrm{du}$ spectre entier qui est limité par un filtre interférentiel isolant la bande $6000-7000 \mathrm{~cm}^{-1}$. D'autres spectres présentant la même résolution ont été pris à travers un autre filtre couvrant la région $7500-8500 \mathrm{~cm}^{-1}$. Chacune des deux traces reproduites ici est en fait une moyenne pondérée de 3 spectres différents. Les 6 spectres utilisés ont été pris au cours. de plusieurs nuits par des conditions atmosphériques variables, parfois franchement médiocres; ils présentent des rapports $s / b$ inégaux, mais pas de différences systématiques supérieures au bruit. Les meilleurs ont reçu, dans le calcul des deux moyennes, des coefficients plus élevés. Cette opération nous paraît justifiée $a$ posteriori, du fait que les différences aléatoires entre les deux traces résultantes présentées ici sont plus faibles qu'entre deux quelconques des traces composantes. L'amélioration du rapport $s / b$ est sensiblement celui que des considérations élémentaires laissent attendre. Le temps total d'observation utilisé pour obtenir les deux traces est égal à 21 heures; nous estimons que par des conditions de transparence excellente, des résultats équivalents auraient pu être obtenus en 12 heures environ.

La figure 16 présente également un spectre lunaire de résolution identique, qui donne les raies solaires et telluriques (principalement dues à $\mathrm{CO}_{2}$ ) dans cette région. La plupart des raies du spectre de Jupiter sont dues à $\mathrm{CH}_{4}$. La figure 17 compare une portion différente du même spectre lunaire avec la région correspondante du spectre solaire tel qu'il est donné par le Mac Math-Hulbert University of Michigan Atlas, dont la résolution est voisine de $0,25 \mathrm{~cm}^{-1}$ dans cette région.

En résumé, nos spectres de Jupiter présentent une résolution comparable à celle de l'Atlas solaire d'usage courant dans le proche I R $\left({ }^{14}\right)$ et 100 fois meilleure que les meilleurs spectres de Jupiter obtenus jusqu'ici.

L'impossibilité pratique d'obtenir de tels résultats par le procédé classique de balayage du spectre est facile à démontrer. Les spectromètres utilisés pour enregistrer les spectres de la figure 13 étaient capables d'utiliser la lumière du disque entier, mais seulement avec le pouvoir de résolution relativement faible qui était demandé. Pour multiplier ce pouvoir de résolution par un facteur $10^{2}$, il aurait fallu consentir une réduction d'un facteur $10^{4}$ sur l'énergie transmise au récepteur : la fente d'entrée n'aurait plus accepté que $1 / 100 \mathrm{du}$ disque planétaire, et la fente de sortie une fraction 100 fois plus faible de l'énergie présente dans le spectre continu. Pour conserver le même rapport signal/bruit $\left({ }^{15}\right)$ il aurait fallu multiplier le

(14) Cette résolution peut naturellement être améliorée - sur le soleil - par nombre de grands spectromètres à réseau existant maintenant.

(15) En théorie, il aurait été possible de réduire simultanément la surface du récepteur. En pratique, il existe une dimension minimum réalisable peu inférieure à celle qui était employée pour les spectres de la figure 13, de sorte que le gain effectif aurait été très modeste, et l'ordre de grandeur de notre résultat ne serait pas modifié. 


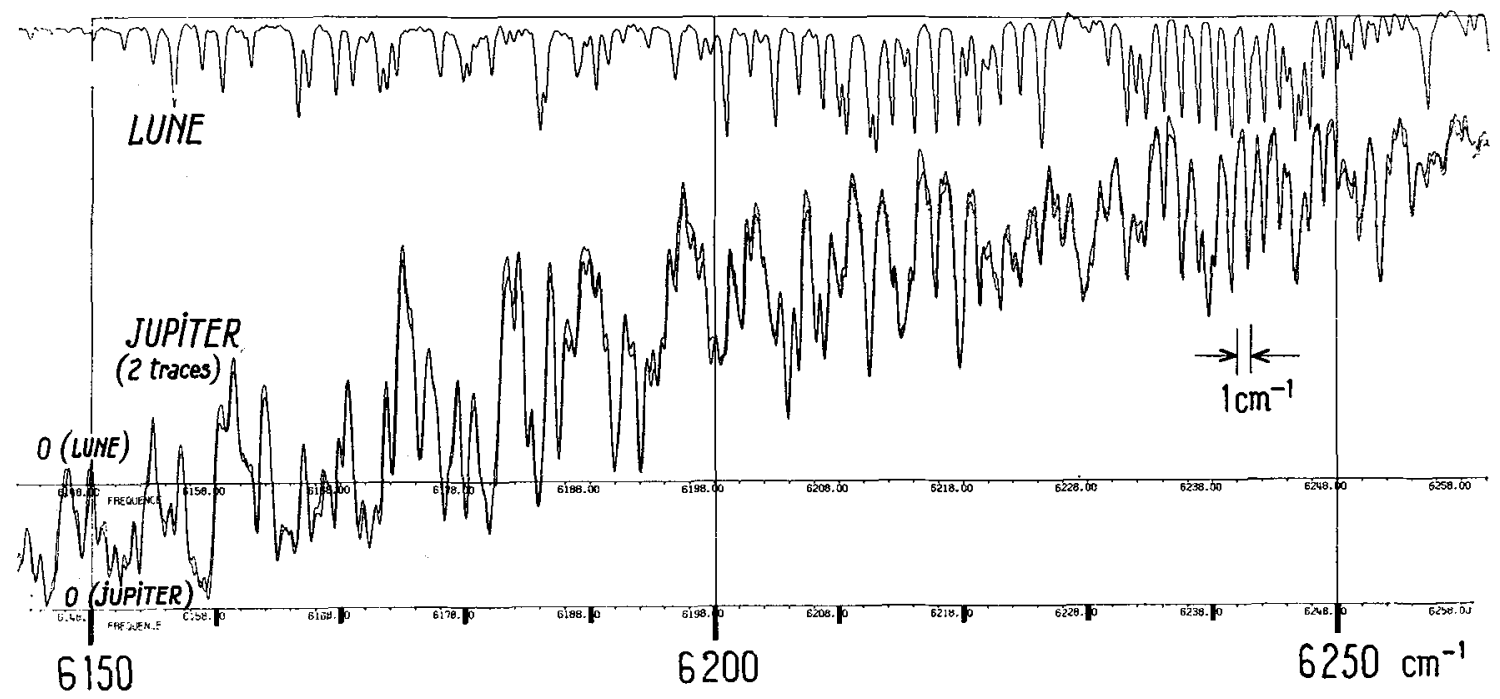

FIg. 16. - Deux spectres de Jupiter. Chaque trace est une moyenne pondérée de 3 enregistrements pris les 1,3 , 4 et 5 janvier 1966. La portion du spectre présentée ici est marquée à gauche des figures 13, 14 et 15. Paramètres : $\Delta \sigma=1400 \mathrm{~cm}^{-1}, \delta \sigma=0,3 \mathrm{~cm}^{-1}$ (avec apodisation due en partie à l'ouverture finie du faisceau traversant l'interféromètre), $M=4700, N=12900$, nombre de franges $\mathrm{Hg}$ entre échantillons $n=5$, rapport $S_{\max } / B_{\text {eff }}=200$ et $S_{\text {moy }} / B_{\text {epf }}=55$, facteur de qualité $q=2,6 \times 10^{5}$. Temps d'observation total $: 21 \mathrm{~h}$. En haut, spectre lunaire de comparaison, résolution identique.
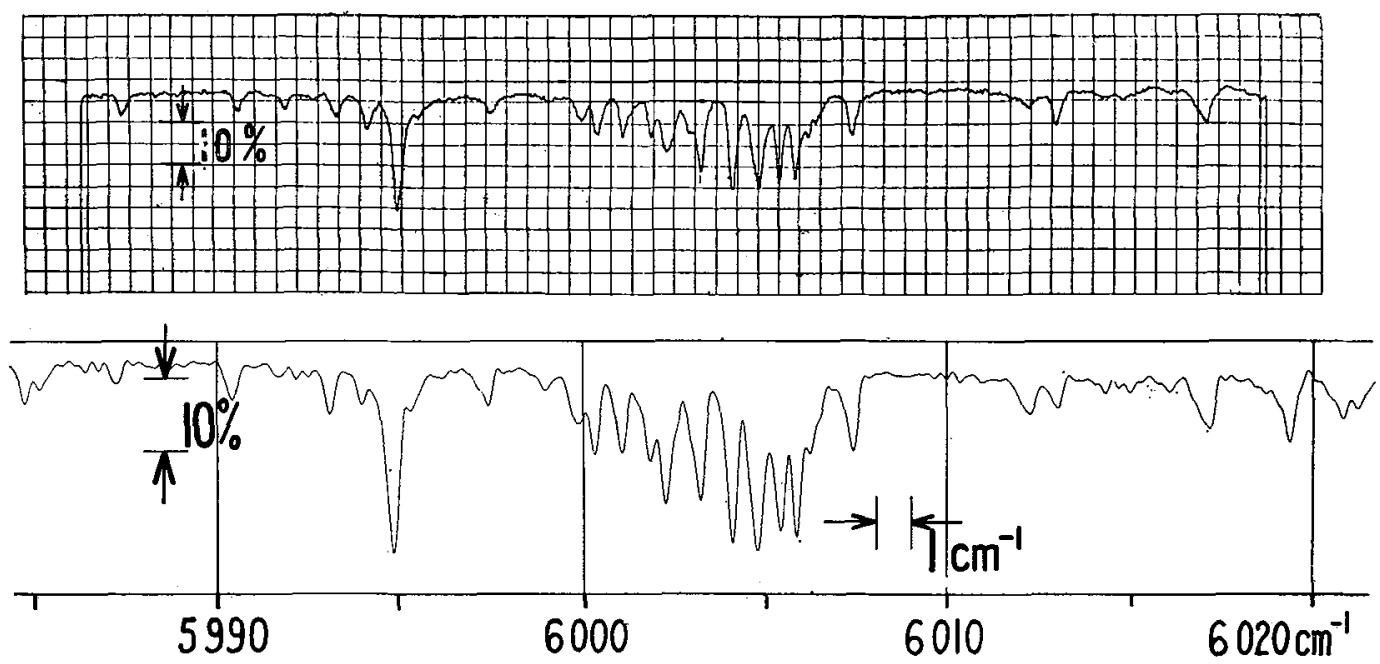

FIG. 17. - Une autre région đu spectre Iunaire de la figure 16, mais tracée ici avec une dispersion double (en bas), comparée à la portion correspondante (en haut) de l'Atlas solaire de l'Université de Michigan, pris au Mont Wilson (altitude $1750 \mathrm{~m}$ ). Les échelles de nombres d'onde sont identiques mais non celles des intensités. Le niveau zéro des enregistrements n'est pas donné, mais une intensité égale à $10 \%$ du fond continu est indiquée. La plupart des raies sont dues au méthane tellurique. Les différences d'absorption s'expliquent par les différences d'altitude (Haute Provence : $650 \mathrm{~m}$ ). La résolution de l'Atlas solaire est légèrement meilleure $\left(0,25 \mathrm{~cm}^{-1}\right.$ contre $0,3 \mathrm{~cm}^{-1}$ ).

temps passé sur chaque élément spectral par $10^{8}$, et pour couvrir le même domaine, contenant maintenant 100 fois plus d'éléments spectraux, multiplier le temps total de mesure par $10^{10}$.

L'emploi d'un appareil utilisant même à la résolution de $0,3 \mathrm{~cm}^{-1}$ tout le flux disponible, par exemple un spectromètre à grille [12], un SISAM [13] ou un interféromètre Fabry-Pérot, aurait permis d'éviter la perte due à la fente d'entrée, donc de multiplier l'énergie tombant sur le récepteur par $10^{2}$ par rapport 


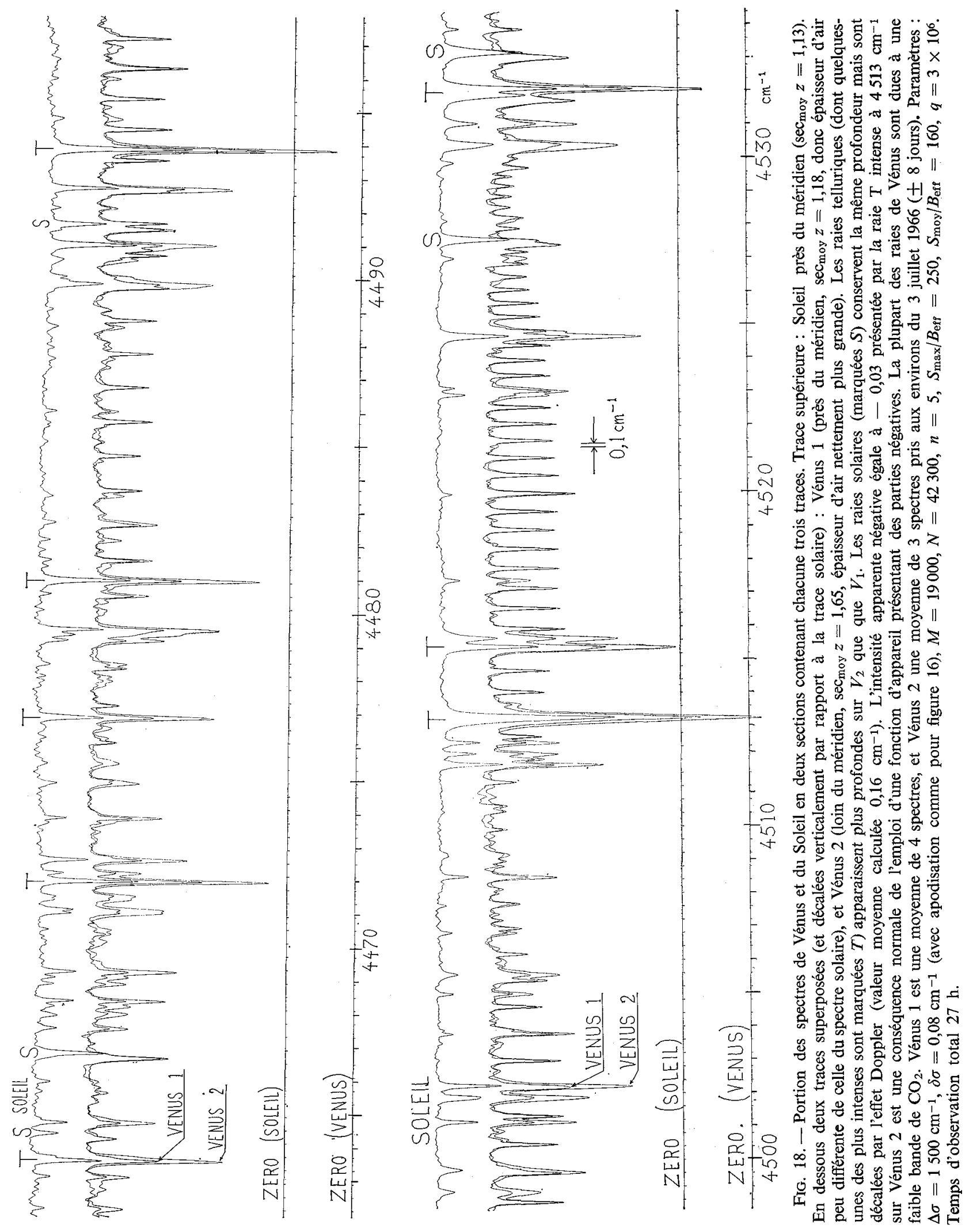


au spectromètre à fente; l'augmentation du temps de mesure aurait alors été seulement égale à $10^{6}$.

Ces résultats illustrent bien l'avantage considérable obtenu dans un problème où le gain dû au facteur multiplex, et celui qui provient de l'accroissement d'étendue jouent simultanément. Ils appellent cependant deux remarques. D'une part, pour les problèmes stellaires, et avec les télescopes actuels, le gain d'étendue à attendre est plus faible. En effet, un spectromètre équipé d'un grand réseau $(L=25 \mathrm{~cm})$ travaillant sous incidence élevée $\left(\varphi=60^{\circ}\right)$ est capable d'accepter un faisceau dont l'étendue est définie par le diamètre du télescope $(D=2 \mathrm{~m})$ et la turbulence atmosphérique (prenons $\varepsilon=2^{\prime \prime}$ pour donner un ordre de grandeur) jusqu'à un pouvoir de résolution $R=20000$. Les étoiles assez brillantes pour permettre de dépasser cette valeur dans l'infrarouge sont très peu nombreuses. Mais la supériorité d'étendue du spectromètre interférentiel pourra néanmoins être exploitée, car la totalité du flux collecté par un télescope de précision optique très médiocre, donnant une tache d'aberration de l'ordre de la minute d'arc serait utilisable. De ce fait, la construction d'un télescope de dimensions nettement supérieures à celles des instruments existants devient concevable.

D'autre part, dans un problème pour lequel l'exploration d'un domaine spectral très étroit est considéré comme suffisant (mesure du profil d'une raie d'absorption), l'avantage à attendre du multiplexage devient faible et il reste permis de songer à utiliser, par exemple, un étalon Fabry-Pérot.

3) VÉNus. La figure 18 illustre les progrès réalisés par rapport à nos premiers spectres de Vénus [1]. La résolution est ici égale à $0,08 \mathrm{~cm}^{-1}$, soit 12 fois meilleure. Le spectre complet, dans une fenêtre de transmission, se présente sous la forme d'une courbe de $75 \mathrm{~m}$ de long à l'échelle de $3 \mathrm{~mm}$ par élément spectral (écheile qui est lisible, mais totalement insuffisante pour utiliser pleinement la précision avec laquelle sont donnés les nombres d'ondes). Cette précision est telle que non seulement l'effet Doppler, mais encore la variation de l'effet Doppler au cours de la période d'observation, sont facilement mesurables (Fig. 19) $\left({ }^{16}\right)$.

(16) Note ajoutée à la correction. La précision de l'échelle des nombres d'onde a depuis été vérifiée en comparant les positions de 38 raies de Vénus dues à $C O$, situées vers $4200 \mathrm{~cm}^{-1}$, aux meilleures valeurs mesurées en laboratoire (D. H. Rank et al., $J$. Mol. Spectr., 1960, 4, 518). La différence moyenne est inférieure à $10^{-3} \mathrm{~cm}^{-1}$ et de l'ordre de l'incertitude sur la correction d'effet Doppler diurne (qui varie de $4 \times 10^{-3} \mathrm{~cm}^{-1}$ pendant la durée d'un enregistrement). La valeur efficace de l'écart quadratique moyen est $2 \times 10^{-3} \mathrm{~cm}^{-1}$.

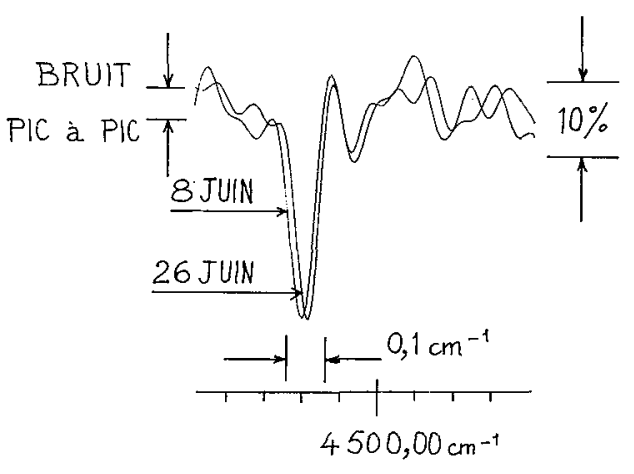

FIG. 19. - Une raie de Vénus extraite de deux spectres pris à 18 jours d'intervalle. Variation de l'effet Doppler calculée $0,016 \mathrm{~cm}^{-1}$. Valeur mesurée sur cette seule raie $0,014 \mathrm{~cm}^{-1}$ avec pour erreur probable $0,005 \mathrm{~cm}^{-1}$. La précision est limitée essentiellement par le bruit ; elle s'améliore lorsqu'une moyenne est prise sur plusieurs raies. Le niveau de bruit est ici plus élevé que celui de la figure 18 car les deux spectres ne sont pas des moyennes. La largeur de raie est plus faible $\left(0,07 \mathrm{~cm}^{-1}\right)$ pour deux raisons : sur la figure 18 les raies de Vénus sont légèrement élargies par la variation d'effet Doppler, et d'autre part l'amélioration du rapport signal/bruit a nécessité l'emploi d'une apodisation plus forte.

Le cas de Vénus dans le domaine de sensibilité des récepteurs à sulfure de plomb, réunit pratiquement les difficultés maximales pour les applications astronomiques de la spectroscopie de Fourier. En effet, il s'agit de l'astre le plus brillant dans la région où il est possible d'atteindre le pouvoir de résolution le plus élevé, et par conséquent les valeurs les plus grandes de $M$ et de $q$. D'autre part, les observations exigeant des temps d'intégration de plusieurs heures sont nécessairement faites en plein jour, donc dans des conditions de turbulence beaucoup plus sévères que les observations de nuit.

Il est juste de remarquer à ce propos que dans le cas d'étoiles relativement faibles, qui représentent évidemment la majorité des objets observables, l'énergie disponible ne permettra jamais de dépasser des pouvoirs de résolution beaucoup plus modestes. Des spectromètres de Fourier astronomiques plus simples que le nôtre, tels que ceux construits par Sinton [14] Mertz [5], Delbouille et Roland [15] et Moroz [16] devraient alors permettre d'obtenir d'aussi bons résultats.

8. Conclusion. - Les principaux résultats que nous pensons avoir établis sont les suivants :

1) La turbulence atmosphérique ne constitue pas un obstacle aux applications astronomiques de la spectroscopie de Fourier. Si elle doit un jour limiter le gain apporté par le principe multiplex, cette limite n'est pas encore atteinte. 
2) Des fluctuations de transparence considérées comme inadmissibles pour la photométrie astronomique par exemple, sont parfaitement tolérables; une transparence très médiocre augmente seulement le temps d'intégration nécessaire.

3) Des spectres obtenus lors de séances différentes sont reproductibles au bruit près; il est facile d'en prendre la moyenne, ce qui améliore le rapport signal/ bruit, et permet d'exploiter pleinement le temps d'observation disponible.

4) La liaison par ligne téléphonique à un grand ordinateur permet d'obtenir sur le spectre les renseignements indispensables dans un délai raisonnable. Seul, un grand ordinateur est capable de traiter le volume considérable des données produites dans le cas d'astres brillants.

La conclusion générale nous paraît être que c'est à présent le temps d'observation disponible seul qui limite l'extension de la spectroscopie infrarouge astronomique et la construction d'un grand collecteur de lumière spécialisé pour ce travail semble justifiée.

Remerciements. - Nous adressons nos remerciements à la Cie IBM France pour avoir, à deux reprises, prêté aimablement le système de transmission 1050.

Messieurs Dornbusch, Mars et Moure se sont dévoués sans compter pour assurer son installation et son entretien et ont largement contribué au succès des observations. Monsieur Collet s'est chargé d'écrire le programme de lecture et décodage des bandes. Madame Gelugne et Monsieur Delouis, à Meudon, ont assuré la réception des bandes. Madame Tualy a surveillé la mise en route des calculs et toute l'équipe des opératrices et opérateurs a assuré l'exploitation en grande partie en dehors des heures normales de service.

A Saint-Michel, Messieurs Bouchareine, Cuisenier,
Michel, Pinard, Roizen et Seguin, ont participé à l'installation et au réglage de l'appareil et aux observations.

Nous remercions aussi pour leur aide indispensable et leur dévouement, Monsieur Lamour et toute l'équipe du télescope de $193 \mathrm{~cm}$.

\section{Bibliographie}

[1] Connes (J.) et (P.), J. Opt. Soc. Amer., 1966, 56, 896.

[2] Pinard (J.), J. Physique, 1967, 28, supplément C2-136.

[3] Fellaett (P.), J. Physique, 1958, 19, 187.

[4] MerTz (L.), J. Physique, 1967, 28, supplément C2-165.

[5] Mertz (L.), J. Physique, 1958, 19, 233.

[6] CONNES (J.), Rev. Opt., 1961, 40, 45, 116, 171, 231.

[7] Cooley (J. W.) et Tukey (J. W.), Math. of Computation, 1965, 19, 297.

[8] Forman (M.), J. Physique, 1967, 28, supplément C2-58.

[9] Kuiper (G.), Mem. Soc. Roy. Sci. Liège, 1964, 9, 365.

[10] Moroz (W. I.), Mém. Soc. Roy. Sci. Liège, 1964, 9, 406.

[11] Delbouille (L.), Roland (G.), Gebbie (H. A.), Mém. Soc. Roy. Sci. Liège, 1964, 9, 125.

[12] Girard (A.), J. Physique, 1967, 28, supplément C2-172.

[13] Verges (J. L.), J. Physique, 1967, 28, supplément C2-176.

[14] Sinton(W. M.), Jour. Quant. Spec. Rad. Transfer, 1963, $8,551$.

[15] Roland (G.), J. Physique, 1967, 28, supplément C2-26.

[16] Moroz (W. I.), Arc. Astr. Ac. Sci., 4 juin 1964, 302.

\section{DISCUSSION}

G. STROKE. - The importance of the advantage of ( stop and go ) motion in your instrument is also related to grating ruling engines. I recently found (in examining a number of gratings ruled on interferometrically controled engines, both ( stop and go », and (continuously ) moving) that (stop and go ) engines (such as Babcok's in Pasadena and Jobin et Yvon, Paris) did tend to produce considerably less ( satellites》 than the continuously moving engines (e. g. such as our M. I. T. engine). 